\title{
Estudio DISCREPA: tratamiento de la enfermedad de Parkinson avanzada y utilización de terapias de segunda línea en Cataluña
}

\author{
Asunción Ávila, Pau Pastor, Lluís Planellas, M. Pilar Gil-Villar, Jorge Hernández-Vara, Ana Fernández-Dorado
}

Introducción. El tratamiento de la enfermedad de Parkinson (EP) es complejo y la instauración de terapias de segunda línea en la EP avanzada sigue siendo controvertida.

Objetivo. Analizar la asistencia de pacientes con EP en Cataluña, con especial atención a la utilización de las terapias de segunda línea en la EP avanzada.

Sujetos y métodos. Encuesta en línea autoadministrada a través de la Societat Catalana de Neurologia a neurólogos de Cataluña que atendían a pacientes con EP.

Resultados. Participaron 72 neurólogos que visitaban una media mensual de 38 pacientes con EP (el 37,3\% con complicaciones motoras). El 86\% preguntaba rutinariamente por complicaciones motoras. Los principales motivos para indicar terapias de segunda línea fueron la discapacidad en off (83,1\%), el impacto de las discinesias (76,9\%), el impacto del tiempo en off $(75,4 \%)$ y el tiempo en off $(73,8 \%)$. El $70 \%$ de los neurólogos declaró limitaciones para instaurar terapias de segunda línea: escasez de recursos en su hospital, falta de tiempo para visitar al paciente o para realizar tareas administrativas y falta de soporte de enfermería (33,3\%). No se utilizan terapias de segunda línea en el $72 \%$ de los pacientes que podrían ser potencialmente candidatos, sobre todo por rechazo del paciente $(37,9 \%)$.

Conclusiones. La mayoría de los neurólogos en Cataluña que visitan pacientes con EP pregunta rutinariamente por complicaciones motoras sin utilizar herramientas específicas. Aunque los neurólogos conocen bien las indicaciones de instauración de terapias de segunda línea, la negativa del paciente, la falta de tiempo y la falta de protocolos asistenciales definidos para derivar a pacientes pueden contribuir a una menor utilización de terapias de segunda línea en la EP avanzada.

Palabras clave. Enfermedad de Parkinson. Estimulación cerebral profunda. Fluctuaciones motoras. Infusión de apomorfina. Infusión intraduodenal de levodopa-carbidopa. Tratamiento invasivo.

\section{Introducción}

La enfermedad de Parkinson (EP) es un trastorno degenerativo progresivo en el que pueden aparecer complicaciones motoras en forma de fluctuaciones motoras (44-80\% de los casos a los 5 años) y discinesias (30-80\% de los casos a los 5-7 años) [1-4]. En los pacientes con complicaciones motoras en los que la terapia habitual con levodopa, agonistas dopaminérgicos, inhibidores de la monoaminooxidasa o de la catecol-O-metiltransferasa fracasa, se utilizan terapias avanzadas o de segunda línea, como infusión intraduodenal de levodopa en gel, infusión continua de apomorfina subcutánea o estimulación cerebral profunda $[1,3,5]$.

La prevalencia mundial de la EP oscila entre 100300 casos/100.000 habitantes según la metodología utilizada, el área geográfica estudiada y los rangos de edad considerados [1,6,7]. Aunque no tenemos datos claros sobre la prevalencia de la EP en Cata- luña, un informe reciente del Servei Català de la Salut [8] muestra una prevalencia en 2017 de 3.632 casos $/ 10^{6}$ habitantes, con una incidencia de 532 casos nuevos $/ 10^{6}$ habitantes, que se ha mantenido estable en el período estudiado (2013-2017). Este informe refiere que en Cataluña, en el año 2017, existían 27.195 pacientes con un diagnóstico de EP y un tratamiento específico para esta enfermedad, aunque en un $22,5 \%$ de los casos, la EP estaba producida por fármacos [8], por lo que supuestamente incluan parkinsonismos farmacológicos. Dentro de la Societat Catalana de Neurologia (SCN), el Grupo de Estudio de Trastornos del Movimiento, con unos 40 miembros, tiene como objetivo promover la mejora de la asistencia, la docencia y la investigación de la EP y otros parkinsonismos dentro del ámbito de Cataluña. Por este motivo, se planteó realizar un estudio de opinión cuyo objetivo principal era conocer la realidad de la asistencia de los pacientes con EP en Cataluña, con especial atención a la EP
Servicio de Neurología; Consorci Sanitari Integral; Hospital Sant Joan Despí Moisès Broggi-Hospital General de I'Hospitalet; Barcelona (A. Ávila). Servicio de Neurología; Unidad de Trastornos del Movimiento; Hospital Universitari Mútua de Terrassa; Terrassa, Barcelona ( P. Pastor). Servicio de Neurología; Hospital Clínic; Barcelona (L. Planellas). Servicio de Neurología; Hospital Arnau de Vilanova; Lleida (M.P. Gil-Villar). Servicio de Neurología; Hospital Universitari Vall d'Hebron; Grupo de Investigación en Enfermedades Neurodegenerativas; Vall d'Hebron Instituto de Investigación; Barcelona (J. Hernández-Vara). Grupo de Estudio de Trastornos del Movimiento; Societat Catalana de Neurologia; Barcelona (A. Ávila, P. Pastor, L. Planellas, M.P. Gil-Villar, J. Hernández-Vara). Adelphi Spain; Barcelona, España (A. FernándezDorado).

Correspondencia:

Dra. Asunción Ávila Rivera. Servicio de Neurología. Consorci Sanitari Integral. Hospital General de I'Hospitalet. Avda. Josep Molins, 29-41. E-08906 L'Hospitalet de Llobregat (Barcelona).

E-mail:

aavila@csi.cat

Conflicto de intereses:

Todos los firmantes han recibido honorarios de Abbvie por el diseño, los comentarios y la promoción de este estudio.

Financiación:

Trabajo financiado por Abbvie.

Aceptado tras revisión externa: 09.09.20.

Cómo citar este artículo: Ávila A, Pastor P, Planellas L, Gil-Villar MP, Hernández-Vara J, Fernández-Dorado A. Estudio DISCREPA: tratamiento de la enfermedad de Parkinson avanzada y utilización de terapias de segunda línea en Cataluña. Rev Neurol 2021; 72: 1-8. doi: 10.33588/ rn.7201.2020181.

(c) 2021 Revista de Neurología 
avanzada, y la indicación e instauración de terapias de segunda línea.

\section{Sujetos y métodos}

Para llevar a cabo un estudio de opinión sobre la EP y las terapias de segunda línea, utilizando metodología cuantitativa, se elaboró una encuesta en línea autoadministrada. La SCN contaba en el momento de la realización de esta encuesta con 621 socios, de los cuales 385 son neurólogos, 143 son médicos de otras especialidades, enfermeras, biólogos, psicólogos, fisioterapeutas, etc., y en 93 miembros se desconoce. Para la difusión de la encuesta en línea y la captación de perfiles deseados, se envió una solicitud de colaboración a todos los socios de la SCN. En el inicio de la encuesta, se seleccionó a los profesionales asociados de la SCN que cumplieran los siguientes criterios de inclusión:

- Neurólogos que ejercían su actividad profesional en Cataluña, en centros públicos, concertados o privados.

- Neurólogos que atendían a pacientes con EP de forma habitual en una consulta de neurología general (CG), una consulta monográfica de trastornos del movimiento (CM) -entendida como consulta experta en trastornos del movimiento- o una unidad de trastornos del movimiento (UTM) -entendida como una unidad con autonomía organizativa formada por un equipo multidisciplinar y con dos o más neurólogos-.

- Se excluyó a residentes, personal de apoyo y personal sanitario sustituto.

Mediante una técnica de muestreo por conveniencia (no probabilístico), participaron 72 neurólogos. Para la recogida de datos se utilizó el programador en línea para diseñar el cuestionario. Se tuvo en cuenta cuidadosamente el orden de las preguntas para evitar que la respuesta a preguntas previas condicionase las respuestas posteriores. Por ello, a modo de ejemplo, se preguntaba sobre la realidad en la práctica clínica antes de preguntar por las limitaciones y los retos, favoreciendo así una reflexión previa en torno estas cuestiones. Tras una conducción del documento entre cinco profesionales sanitarios del entorno del estudio, se procedió a la apertura del cuestionario el 22 de mayo de 2019. Desde esa fecha, se contactó con individuos estratégicos para su difusión. El 31 de agosto de 2019 se cerró la encuesta y se comprobó que todos los participantes cumplían criterios de inclusión y que no había inconsistencia en sus respuestas.
Se realizó un análisis estadístico descriptivo de las variables estudiadas. Se describieron las características de los participantes mediante tablas de frecuencias para las variables de tipo nominal, y medidas de tendencia central y dispersión para las variables continuas. Para los modelos bivariantes se utilizaron los test ANOVA y chi cuadrado. Para todos los análisis se utilizó el programa SPSS v. 15.0.

En cuanto a los aspectos éticos, el cuestionario se cumplimentó de forma voluntaria y anónima por el profesional manteniendo la confidencialidad y el secreto estadístico, de acuerdo con el código deontológico de Farmaindustria, el código de conducta de ESOMAR y AEDEMO, así como por el Reglamento (UE) 2016/679, de 27 de abril de 2016, o el Reglamento General de Protección de Datos y la normativa nacional vigente.

\section{Resultados}

\section{Caracterización de la muestra}

El cuestionario fue contestado por 72 neurólogos (el 6\% de Lleida, el 11\% de Girona, el 11\% de Tarragona y el 72\% de Barcelona). El 75\% de los neurólogos tenía más de cinco años de experiencia tras finalizar la especialidad y el 69\% trabajaba en un centro público. Los neurólogos participantes visitaban a sus pacientes con EP en una CG en el $54 \%$ de los casos, en una CM en el $22 \%$ y en una UTM en el $24 \%$.

Los neurólogos encuestados visitaban una media de $38 \pm 34,7$ pacientes con EP/mes, de los cuales en $14 \pm 15,2$ pacientes/mes se detectaban complicaciones motoras $(37,3 \%)$. Curiosamente, no hallamos diferencias estadísticamente significativas en el porcentaje de pacientes con complicaciones motoras según el tipo de consulta (CG, 34,1\%; CM, 37,4\%; UTM, 44,5\%; $p=0,265$ ), aunque el número total de pacientes con EP visitados mensualmente y, por tanto, el número total de pacientes con complicaciones motoras eran significativamente superiores en las UTM y en las CM respecto a las CG (Tabla I).

\section{Motivos de derivación a especialistas en trastornos del movimiento}

Los principales motivos para derivar a un paciente a un especialista en EP, teniendo en cuenta que podía haber más de un motivo de derivación, y excluyendo a los neurólogos que trabajaban en una UTM que no derivan a especialistas en trastornos del movimiento, fueron: EP en paciente joven, difícil control de la enfermedad y sospecha de EP familiar. 
Otros motivos frecuentes eran si el paciente presentaba complicaciones motoras o si existía un parkinsonismo no filiado o atípico (Fig. 1).

\section{Detección de complicaciones motoras}

El 86\% de los neurólogos preguntaba de manera rutinaria a los pacientes con EP sobre la presencia de complicaciones motoras, un $37,1 \%$ utilizaba diarios del paciente y un 22,6\% usaba alguna escala o cuestionario. No hubo diferencias estadísticamente significativas en el porcentaje de pacientes declarados con complicaciones motoras entre neurólogos que preguntaban rutinariamente por ellas y los que no lo hacían.

Los neurólogos encuestados declararon no conseguir un buen control de las complicaciones motoras en el 33,15\% de sus pacientes, sin existir diferencias estadísticamente significativas entre los distintos tipos de consulta (CG, 39,7\%; CM, 22,6\%; UTM, $28,5 \% ; p=0,069)$. Cuando analizamos cómo se procedía con estos pacientes con control subóptimo de las complicaciones motoras, el 36,6\% de neurólogos continuaban ellos mismos el seguimiento, el 16,9\% los derivaba a un especialista en EP del mismo hospital y un 46,5\% los derivaba a una UTM de otro hospital. Obviamente, existían diferencias en las acciones realizadas por los neurólogos ante pacientes con mal control de complicaciones motoras según el tipo de consulta en el que se ejercía (Tabla II).

\section{Terapia avanzada o de segunda línea}

\section{Actitud ante el paciente con control subóptimo de complicaciones motoras}

Del 36,6\% de los neurólogos que continuaron encargándose del seguimiento de pacientes con un control subóptimo de las complicaciones motoras, el $88,5 \%$ se planteó en algún momento iniciar una terapias de segunda línea (70\% entre los neurólogos de CG y $100 \%$ entre los neurólogos de CM y UTM; $p=0,02)$. Los neurólogos referían un tiempo medio en plantearse una terapias de segunda línea de 5,4 meses (mediana: 6 meses) y un tiempo medio en realizar la derivación a terapias de segunda línea de 4,5 meses (mediana: 3,5 meses). Considerando el número total de pacientes con control subóptimo de las complicaciones motoras respecto al número de terapias de segunda línea implementadas (véase el siguiente apartado), detectamos que sólo un $28 \%$ de estos pacientes recibe una terapia de segunda línea, por lo que podría haber hasta un $72 \%$ de candidatos potenciales que no recibe una terapia de segunda línea (véase más adelante para los motivos).
Figura 1. Motivos para derivar a un paciente a especialistas en trastornos del movimiento según los neurólogos encuestados.

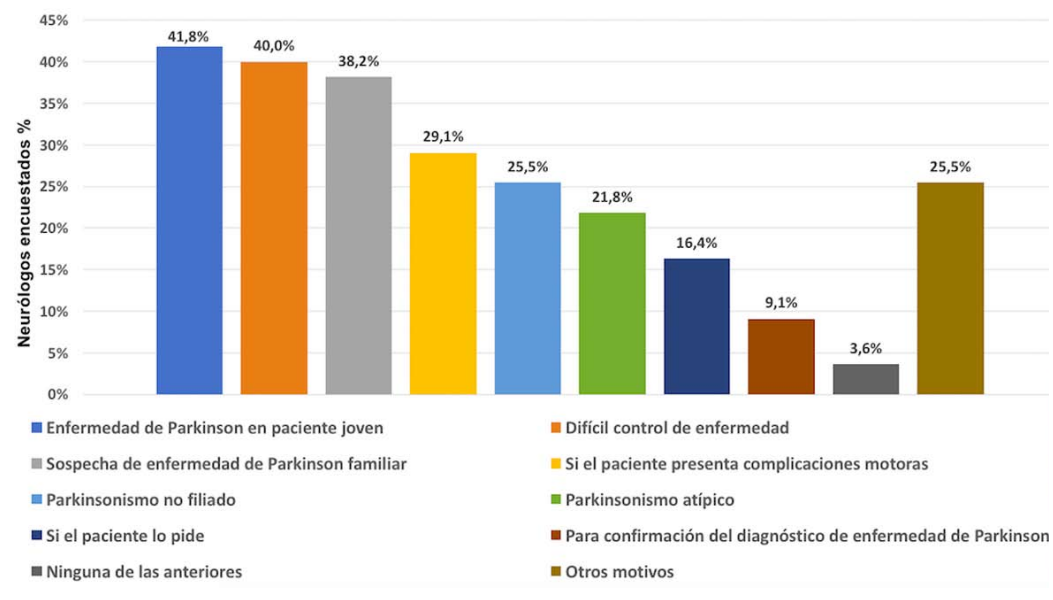

Tabla I. Pacientes con enfermedad de Parkinson y complicaciones motoras visitados mensualmente en los diferentes tipos de consulta neurológica.

\begin{tabular}{|c|c|c|c|c|}
\hline & & $\begin{array}{c}\text { Pacientes } \\
\text { visitados/mes }\end{array}$ & $\begin{array}{c}\text { Pacientes con } \\
\text { complicaciones } \\
\text { motoras/mes }\end{array}$ & $\begin{array}{c}\text { Pacientes con } \\
\text { complicaciones } \\
\text { motoras (\%) }\end{array}$ \\
\hline \multirow{3}{*}{$\begin{array}{l}\text { Consulta de neurología } \\
\text { general }(n=39)\end{array}$} & Media $\pm D E$ & $20 \pm 25,8$ & $6 \pm 9,9$ & \multirow{3}{*}{34,1} \\
\hline & Mediana & 15 & 3 & \\
\hline & IC 95\% & $12-28$ & $3-9$ & \\
\hline \multirow{3}{*}{$\begin{array}{l}\text { Consulta monográfica } \\
\text { de trastornos del } \\
\text { movimiento }(n=16)\end{array}$} & Media $\pm \mathrm{DE}$ & $49 \pm 32,3$ & $19 \pm 16,8$ & \multirow{3}{*}{37,4} \\
\hline & Mediana & 45 & 15 & \\
\hline & IC 95\% & $33-65$ & $11-27$ & \\
\hline \multirow{3}{*}{$\begin{array}{l}\text { Unidad de trastornos } \\
\text { del movimiento }(n=17)\end{array}$} & Media \pm DE & $69 \pm 28,2$ & $29 \pm 10,7$ & \multirow{3}{*}{44,5} \\
\hline & Mediana & 60 & 28 & \\
\hline & IC 95\% & $56-83$ & $24-34$ & \\
\hline
\end{tabular}

DE: desviación estándar; IC 95\%: intervalo de confianza al 95\%.

Los principales motivos para indicar una terapia de segunda línea fueron la discapacidad en off $(83,1 \%)$, el impacto de las discinesias $(76,9 \%)$, el impacto del tiempo en off $(75,4 \%)$ y el tiempo en off $(73,8 \%)$. Otros motivos menos frecuentes fueron las fluctuaciones no motoras incapacitantes $(64,4 \%)$, el tiempo con discinesias (52,3\%), la mala calidad del 
Tabla II. Acciones que se realizan según el tipo de consulta ante un paciente con control subóptimo de las complicaciones motoras.

\begin{tabular}{lccc}
\hline & $\begin{array}{c}\text { Pacientes } \\
\text { que continúa } \\
\text { tratando usted } \\
\text { mismo }\end{array}$ & $\begin{array}{c}\text { Pacientes que } \\
\text { deriva a otro especialista } \\
\text { en Parkinson de } \\
\text { su mismo centro }\end{array}$ & $\begin{array}{c}\text { Pacientes que deriva } \\
\text { a otro centro hospitalario } \\
\text { con unidad de trastornos } \\
\text { del movimiento }\end{array}$ \\
\hline $\begin{array}{l}\text { Consulta de neurología } \\
\text { general }(n=38)\end{array}$ & $26,3 \%$ & $26,3 \%$ & $47,4 \%$ \\
\hline $\begin{array}{l}\text { Consulta monográfica } \\
\text { de trastornos del } \\
\text { movimiento }(n=16)\end{array}$ & $31,3 \%$ & $6,2 \%$ & $62,5 \%$ \\
$\begin{array}{l}\text { Unidad de trastornos } \\
\text { del movimiento }(n=17)\end{array}$ & $64,7 \%$ & $5,9 \%$ & $29,4 \%$ \\
\hline
\end{tabular}

on (43\%), el número de tomas de levodopa (40\%) y la necesidad de ayuda para actividades de la vida diaria (38,5\%). Ningún neurólogo consideró candidato a terapias de segunda línea a pacientes con EP y demencia.

\section{Instauración de terapia de segunda línea}

Mientras que el 47,5\% de neurólogos encuestados instauró alguna vez terapias de segunda línea y derivaba a otros neurólogos para la decisión de su implantación, un 29,5\% no instauró ninguna terapia de segunda línea y sólo derivaba a los pacientes, y un 11,5 \% sólo instauraba terapias de segunda línea, pero nunca derivaba a otro neurólogo para instaurarlas. Es importante resaltar que el 22,6\% de los neurólogos de una CG encuestados no instauraba terapias de segunda línea y tampoco derivaba a los pacientes para ello (Fig. 2).

El número medio de estimulaciones cerebrales profundas instauradas fue de $8 \pm 6,7$ al año (rango 4-12) sobre una muestra de 11 neurólogos. En cuanto a las infusiones de apomorfina, se instauró una media anual de $3 \pm 2,5$ (rango 2-4) sobre 33 neurólogos; y a las infusiones intraduodenales de levodopa, una media anual de $5 \pm 6,9$ (rango 2-8) sobre 21 neurólogos. Por último, en una muestra de 46 neurólogos se derivaba una media de $5 \pm 3$,7 pacientes/ año a terapias de segunda línea (rango 3-6).

Entre las terapias de segunda línea que se instauraron en el mismo centro, el 55,7\% de los neurólogos instauraba infusión continua de apomorfina subcutánea, el 36,1\% instauraba infusión intraduodenal de levodopa en gel y el 18\% realizaba estimulación cerebral profunda en su propio hospital. Por el contrario, la terapia de segunda línea para la que se derivaba en mayor porcentaje a otro hospital era la estimulación cerebral profunda $(68,9 \%)$, seguida de la infusión
Figura 2. Actitud de los neurólogos frente a la instauración de terapias de segunda línea según el tipo de consulta. CG: consulta de neurología general; $\mathrm{CM}$ : consulta monográfica de trastornos del movimiento; UTM: consulta en una unidad de trastornos del movimiento.

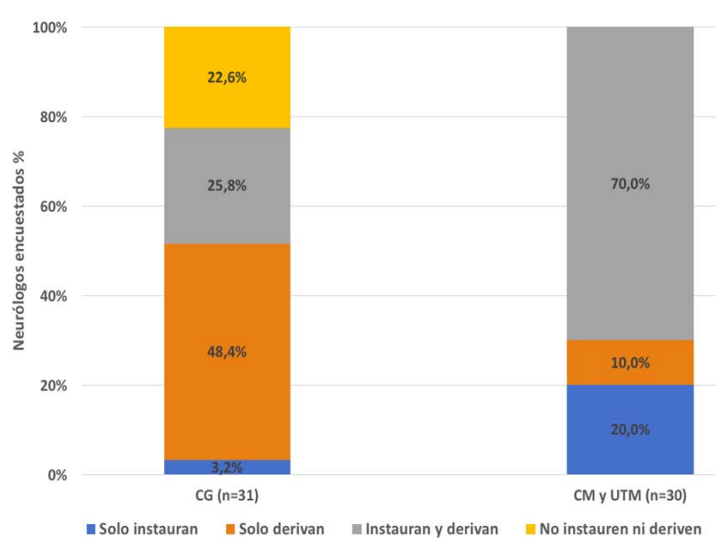

intraduodenal de levodopa en gel $(47,5 \%)$ y, por último, la infusión continua de apomorfina subcutánea $(31,7 \%)$. Evidentemente, existían diferencias entre los neurólogos encuestados según el tipo de consulta en la que trabajaban, de modo que, desde la mayoría de las CG, los pacientes se derivaban a otro hospital para realizar cualquier terapia de segunda línea, mientras que desde las CM y las UTM, se derivaban sobre todo para cirugía (Fig. 3).

\section{Limitaciones para instaurar una terapia de segunda línea}

El 70\% declaró tener alguna limitación para la instauración de terapias de segunda línea, y las principales causas fueron la falta de recursos en su centro hospitalario $(38,1 \%)$, la falta de tiempo para visitar al paciente $(33,3 \%)$, la falta de soporte de enfermería especializada $(33,3 \%)$ y la falta de tiempo para las tareas administrativas requeridas (31\%). Otras limitaciones fueron la falta de presupuesto $(21,4 \%)$, la ausencia de un circuito de derivación $(14,3 \%)$ y el desconocimiento sobre terapias de segunda línea o circuitos existentes para ello (9,5\%). Sólo un 7,1\% y $2,4 \%$ de los neurólogos consideraron como limitación para instaurar terapias de segunda línea los efectos secundarios posibles o su escaso beneficio terapéutico, respectivamente.

Según el tipo de consulta, la principal limitación para instaurar una terapia de segunda línea para los neurólogos de una CG era la falta de tiempo, tanto para interrogar o examinar a los pacientes (para el $37,5 \%$ de los neurólogos) como para realizar las ta- 
reas administrativas (37,5\%). Destacamos que un 25\% de los neurólogos de una CG refería no disponer de un circuito de derivación para terapias de segunda línea. Por otro lado, los neurólogos de una CM consideraban que su principal limitación era la falta de recursos en su centro (50\%), mientras que a los neurólogos de una UTM les limitaba sobre todo la falta de soporte de enfermería (50\%).

\section{Motivos por los cuales se infrautilizan terapias de segunda línea}

Considerando el número de pacientes con control subóptimo de sus complicaciones motoras respecto al número de terapias de segunda línea implementadas, detectamos que sólo un $28 \%$ de estos pacientes recibe una terapia de segunda línea. Por tanto, un $72 \%$ de los pacientes que en principio podrían ser candidatos a terapias de segunda línea por falta de control de síntomas no llega a ser tratado, y el principal motivo $(37,9 \%)$ es el rechazo por parte del paciente o sus familiares. Otros motivos de no tratamiento con terapias de segunda línea se atribuyeron al sistema de salud o a los propios profesionales $(62,1 \%)$, como es el retraso en el proceso de derivación $(27,6 \%)$, la falta de formación de los profesionales $(17,2 \%)$, la falta de recursos del centro $(13,8 \%)$, la limitación del presupuesto hospitalario $(13,8 \%)$, la falta de tiempo $(10,3 \%)$ y la falta de soporte de enfermería (10,3\%). No hubo diferencias estadísticamente significativas entre neurólogos de CG, GM y UTM.

\section{Recursos disponibles para \\ la terapia de segunda línea}

El 75,9\% refería disponer de servicio de endoscopia; el 70,7\%, de neuropsicología; el 67,2\%, de enfermería; y el 50\%, de neurocirugía. Sólo un 31\% disponía de un protocolo de derivación para terapias de segunda línea aprobado y establecido. Si analizamos por separado los recursos disponibles para los neurólogos de CG y los de CM y UTM, existían diferencias estadísticamente significativas $(p=0,034)$, sobre todo en cuanto a la disponibilidad de neuropsicología (65,5\% en CG frente a 75,9\% en CM/UTM), el servicio de neurocirugía competente $(41,4 \%$ en CG frente a $58,6 \%$ el CM/UTM) y el protocolo de derivación (13,8\% en CG frente a 48,3\% en CM/UTM).

\section{Demandas o retos para mejorar la instauración de una terapia de segunda línea}

La última parte del cuestionario preguntaba soluciones para mejorar la identificación y el manejo de los pacientes con EP y complicaciones motoras mal controladas, así como para optimizar la instauración de terapias de segunda línea. El 68,5\% de los neurólogos
Figura 3. Instauración o derivación de las diferentes terapias de segunda línea (TSL) según los tipos de consulta. CG: consulta de neurología general; CM: consulta monográfica de trastornos del movimiento; UTM: consulta en una unidad de trastornos del movimiento.

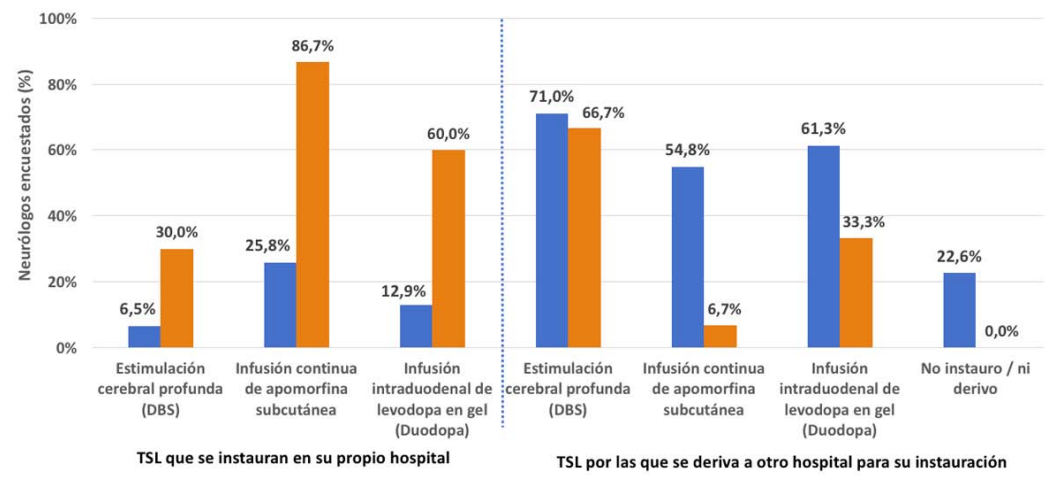

consideró que disponer de más tiempo en la consulta por paciente facilitaría el control de la enfermedad y la instauración de terapias de segunda línea, y era la principal necesidad para el 52,9\%. Otras necesidades mencionadas por orden de prioridad fueron disponer de soporte de enfermería $(44,4 \%)$, reducir las tareas administrativas para instaurar terapias de segunda línea $(42,6 \%)$, formar a los profesionales en terapias de segunda línea $(40,7 \%)$, disponer de más CM en los centros (38,9\%), introducir herramientas de detección de EP avanzada en la práctica clínica habitual $(38,9 \%)$, proporcionar más soporte al paciente o cuidador durante y después de instaurar terapias de segunda línea (38,9\%), disponer de un centro de referencia de rápida derivación $(33,3 \%)$ y reducir las limitaciones presupuestarias (33,3\%). Si analizamos estas necesidades agrupando a los neurólogos de CG, por un lado, y a los de CM y UTM, por otro, la necesidad principal continuaba siendo el incremento del tiempo de consulta.

\section{Discusión}

La EP es una enfermedad neurodegenerativa progresiva en la que se desarrollan complicaciones motoras, en forma de fluctuaciones y discinesias, al cabo de tres a cinco años desde el inicio del tratamiento. Los pacientes alternan períodos de buen y mal control sintomático, considerando que es una EP avanzada cuando la terapia convencional no proporciona un control adecuado de los síntomas [2,9]. La presencia de otra sintomatología motora y no motora, como alteración del equilibrio, disfagia y di- 
sartria, y demencia, disautonomía, dolor o alteraciones psiquiátricas, complica la EP avanzada [2,3].

El tratamiento de la EP inicial es relativamente fácil y está bien establecido [1,4,10]. Sin embargo, el tratamiento de la EP avanzada es más complejo y, aunque se ha tratado de consensuar, la instauración de terapias de segunda línea sigue siendo objeto de debate $[1,3,5]$ y la bibliografía sobre la opinión de especialistas sobre este tema en la práctica diaria es escasa.

A través de este cuestionario, vemos que los neurólogos que ejercen su actividad asistencial en $\mathrm{Ca}$ taluña y que están implicados en la atención al paciente con EP desde una consulta tanto general como específica consideran que un $37,3 \%$ de sus pacientes con EP presentaba complicaciones motoras, fluctuaciones o discinesias. Este porcentaje aumentaba hasta el $44,5 \%$ en las UTM, donde sin duda se atiende a pacientes con EP más avanzada. El porcentaje total es similar al que se recoge en otros estudios [3,11,12]. Aunque un 86,1\% de los neurólogos encuestados preguntaba de forma rutinaria a sus pacientes por las complicaciones motoras, la mayoría no utilizaba herramientas específicas para detectarlas. Un mayor uso de cuestionarios, escalas o diarios de fluctuaciones y discinesias probablemente hubiera incrementado la detección de complicaciones motoras.

En nuestro estudio, los neurólogos consideraron que en el 33,15\% de los pacientes con complicaciones motoras existía un mal control de éstas. Sin embargo, aunque las dificultades en el buen control de la enfermedad aparecían como uno de los principales motivos para derivar a los pacientes desde las CG a las CM o UTM, la aparición de complicaciones motoras sólo motivaba la derivación para el $27,8 \%$ de los neurólogos participantes. Hay que tener en cuenta que uno de los principales motivos de derivación a un especialista en trastornos de movimiento era que el paciente con EP fuera joven, ya que éstos son los que presentarán más frecuente y precozmente complicaciones motoras incapacitantes. Por lo tanto, si consideramos que al grupo de pacientes jóvenes se le deriva tras realizar el diagnóstico de EP a una CM o UTM, cuando con la evolución presente complicaciones motoras, ya lo estarán controlando neurólogos especialistas y, por tanto, no se le va a derivar por este motivo.

Por otro lado, a pesar de detectar un control subóptimo de las complicaciones motoras, el 26,3\% de los neurólogos de CG y el 31,3\% de los neurólogos de CM continúan tratando ellos mismos al paciente, y por diferentes motivos no se remite a una CM o a una UTM.
En relación con las terapias de segunda línea, prácticamente la mitad de los neurólogos encuestados que atienden a pacientes con EP instauraba alguna terapia de segunda línea (sobre todo, infusión continua de apomorfina) y derivaba para otras (sobre todo, cirugía). Llama la atención que un 22,6\% de los neurólogos de una CG no sólo no instauraba ninguna terapia de segunda línea, sino que tampoco derivaba a los pacientes parkinsonianos potencialmente candidatos a terapias de segunda línea. La mayoría de las CM y las UTM instaura infusión continua de apomorfina subcutánea e infusión intraduodenal de levodopa en gel. Sin embargo, un 29,4\% de los neurólogos de una UTM deriva a otra UTM, sobre todo para cirugía y, en menor medida, para la instauración de una infusión intraduodenal de levodopa en gel. Después del plan de reordenación de la cirugía de los trastornos del movimiento en Cataluña [13], ésta se realiza en dos UTM expertas formadas por cuatro hospitales ubicados en la provincia de Barcelona, por lo que los pacientes se derivan desde el resto de los hospitales de Cataluña.

La mayoría de los neurólogos participantes, independientemente de que trabajasen en CG, CM o UTM, conocía bien las indicaciones de terapias de segunda línea, al coincidir en que la discapacidad en off es la característica más relevante, seguida de las discinesias y el tiempo en off, y siempre en pacientes con EP sin demencia. En este aspecto, aunque el diagnóstico de EP avanzada es un requisito para considerar cualquiera de las tres terapias de segunda línea, en ocasiones, están contraindicadas precisamente por la existencia de datos clínicos de enfermedad más avanzada, como la demencia. Sin embargo, aunque ningún neurólogo consideró candidato a terapia de segunda línea a un paciente con demencia, la instauración de infusión intraduodenal de levodopa tiene una contraindicación relativa en el caso de demencia grave, pero no en pacientes con EP avanzada y demencia moderada o leve [5]. Otro punto que hay que destacar es que solo el $43,1 \%$ consideraba la mala calidad del on relevante para instaurar una terapia de segunda línea.

Aunque la gran mayoría de los neurólogos $(70 \%$ entre los neurólogos de CG y 100\% entre los neurólogos de CM y UTM) se planteaba en algún momento una terapia de segunda línea ante un mal control de las complicaciones motoras, pocos pacientes de los potencialmente candidatos (28\%) acababan siendo tratados con una terapia de segunda línea, y el rechazo por parte del paciente o de los familiares era el principal motivo de que no se tratase. Otros motivos se atribuyeron al sistema de salud o a los 
propios profesionales: retraso en el proceso de derivación, falta de formación de los profesionales, falta de recursos del centro, limitación del presupuesto hospitalario, falta de tiempo y falta de soporte de enfermería. Recientemente, se han presentado datos sobre el estudio PARADISE [14-16], que analiza de forma prospectiva la EP avanzada en hospitales del sistema público de salud español. Aunque por su diferente metodología es poco valorable compararlo con nuestros resultados, el estudio PARADISE $[14,15]$ refiere que sólo un $19,5 \%$ de los pacientes con EP avanzada acababa con terapias de segunda línea, y los principales motivos del no tratamiento eran diferentes a los nuestros: la estabilidad clínica del paciente con EP avanzada (36,6\%), el no haberse valorado la posibilidad de momento $(25,5 \%)$ y la contraindicación de las tres terapias de segunda línea $(15,4 \%)$. El rechazo a la terapia de segunda línea por parte del paciente o el cuidador sólo se consideró en un 13,4\% de los casos, muy lejos de nuestro $37,9 \%$. No sabemos explicar las diferencias en este porcentaje, aunque en el rechazo a la instauración de una terapia de segunda línea por parte del paciente o su entorno influyen factores como la falta de tiempo en la consulta o la falta de habilidades en la comunicación médico-paciente. $\mathrm{Si}$ se dispusiera de más tiempo en las consultas y más personal de enfermería para explicar con más detenimiento las terapias de segunda línea, se podrían resolver todas las dudas de los pacientes y quizá el porcentaje de pacientes que accedería a terapias de segunda línea podría ser mayor. Otros motivos atribuidos al propio sistema de salud o a los profesionales podrían también, sin duda, mejorarse. Sólo una tercera parte de los neurólogos encuestados disponía de un protocolo de derivación de pacientes aprobado y establecido para instaurar terapias de segunda línea, pero este porcentaje era todavía más bajo $(13,8 \%)$ entre los neurólogos de CG.

Por último, cabe destacar que la principal demanda de los neurólogos encuestados, independiente de que trabajen en CG, CM o UTM, era la necesidad de incrementar el tiempo de consulta dedicado al paciente con EP para mejorar la atención al paciente y facilitar la instauración de terapias de segunda línea. Entre los neurólogos de CG también era importante disponer de más CM en los centros, introducir herramientas de detección de EP avanzada en la práctica clínica habitual y disponer de un centro de referencia de rápida derivación. En cambio, los neurólogos de CM y UTM deseaban disponer de una enfermera especializada, proporcionar más soporte al paciente con terapias de segunda línea y reducir las limitaciones presupuestarias.
Una limitación de este estudio podría ser el bajo número de neurólogos que contestaron la encuesta, teniendo en cuenta que se envía a todos los socios de la SCN. Sin embargo, consideramos que puede ser una muestra representativa del total de neurólogos que ejercen en Cataluña (311 en el año 2013 [17], cuando la SCN contaba con más de 350 socios), al incluir neurólogos de las cuatro provincias catalanas de forma proporcional a su lugar de ejercicio y neurólogos de distintos tipos de consultas (CG, CM y UTM) en número suficiente. Además, la mayoría de los socios de la SCN no podían participar en la encuesta, al requerirse una atención regular a pacientes con EP.

Otra limitación de este estudio es la dificultad que supone responder con exactitud a algunas preguntas del cuestionario, como las relacionadas con el número mensual de pacientes con EP visitados, los porcentajes de pacientes con complicaciones motoras, el número de terapias de segunda línea derivadas o el tiempo que tardamos en derivar a un paciente. Un estudio prospectivo sobre la actitud ante un paciente con EP avanzada y la instauración de terapias de segunda línea daría datos más fiables. Por último, se podía haber incluido en la encuesta alguna pregunta para conocer el peso de las complicaciones no motoras en la decisión de instauración de terapias de segunda línea. Es bien conocido que los síntomas no motores de la EP pueden llegar a ser tan incapacitantes como los síntomas motores $y$, en ocasiones, van a condicionar la instauración de determinadas terapias de segunda línea.

En conclusión, nuestro estudio confirma que la mayoría de los neurólogos que trabajan en Cataluña y están implicados en la atención a pacientes con EP pregunta de forma rutinaria por las complicaciones motoras, aunque no utiliza herramientas específicas para su detección. En general, ante el control subóptimo de las complicaciones motoras, aunque los neurólogos encuestados conocen correctamente las indicaciones de instauración de terapias de segunda línea, la negativa del paciente, por un lado, y la falta de tiempo en las consultas, la escasez de enfermería especializada y la falta de protocolos para derivar a los pacientes, por otro, contribuyen a que se instauren menos terapias de segunda línea. Es necesario aumentar el tiempo de la visita del paciente con EP no sólo para favorecer la anamnesis, sino para incrementar la utilización de herramientas de detección de complicaciones motoras, explicar con más detenimiento las terapias de segunda línea y mejorar su instauración. Además, deberíamos establecer protocolos de derivación de pacientes desde 
las consultas generales a las específicas, aumentar la enfermería especializada, proporcionar más soporte al paciente con terapias de segunda línea y reducir las limitaciones presupuestarias.

\section{Bibliografía}

1. Gironell A, De Fàbregues $\mathrm{O}$, Compta $\mathrm{Y}$, coords. Protocol de diagnòstic i tractament de la malaltia de Parkinson. Guies Médiques de la Societat Catalana de Neurologia. Actualització 2016. Barcelona: SCN; 2016.

2. Kulisevsky J, Luquin MR, Arbelo JM, Burguera JA, Carrillo F, Castro A, et al. Enfermedad de Parkinson avanzada. Características clínicas y tratamiento (parte I). Neurologia 2013; 28: 503-21.

3. De Fàbregues O, Gironell A, Roselló-Jiménez D, Regueras E. Unidades de trastornos del movimiento y tratamiento de las fluctuaciones motoras de la enfermedad de Parkinson avanzada. Rev Neurol 2017; 65: 396-404.

4. Olanow CW, Watts RL, Koller WC. An algorithm (decision tree) from the management of Parkinson's disease: treatment guidelines. Neurology 2001; 56 (Suppl 5): S1-88.

5. Kulisevsky J, Luquin MR, Arbelo JM, Burguera JA, Carrillo F, Castro A, et al. Enfermedad de Parkinson avanzada. Características clínicas y tratamiento (parte II). Neurología 2013; 28: 558-83.

6. García-Ramos R, López-Valdés E, Ballesteros L, Jesús S, Mir P. Informe de la Fundación del Cerebro sobre el impacto social de la enfermedad de Parkinson en España. Neurologia 2016; 31: 401-13.

7. Benito-León J, Epidemiología de la enfermedad de Parkinson en España y su contextualización mundial. Rev Neurol 2018; 66: $125-34$.

8. Servei Català de la Salut, Unitat d'Informació i Coneixement, Pla Director Sociosanitari (Cataluña). Persones amb malaltia de Parkinson: Cataluña 2013-2017 (31/2019). URL: http:// scientiasalut.gencat.cat/handle/11351/4061. [30.04.2019].

9. Martínez-Martín P, Kulisevsky J, Mir P, Tolosa E, García-
Delgado P, Luquin MR. Validation of a simple screening tool for early diagnosis of advanced Parkinson's disease in daily practice: the CDEPA questionnaire. NPJ Parkinsons Dis 2018 4: 20

10. Arbelo-González JM, coord. Guía oficial de práctica clínica en Parkinson. Guías Diagnósticas y Terapéuticas de la Sociedad Española de Neurología. Madrid: SEN; 2016.

11. Bjornestad A, Forsaa EB, Pedersen KF, Tysnes OB, Larsen JP, Alves G. Risk and course of motor complications in a populationbased incident Parkinson's disease cohort. Parkinsonism Relat Disord 2016; 22: 48-53.

12. Scott NW, Macleod AD, Counsell CE. Motor complications in an incident Parkinson's disease cohort. Eur J Neurol 2016; 23: 304-12.

13. Servei Català de la Salut. Reordenació de la cirurgia dels trastorns del moviment (03/2018). URL: http://scientiasalut. gencat.cat/handle/11351/3640. [08.10.2018].

14. Martínez-Castrillo JC, Martínez-Martín P, Burgos A, Arroyo G, García N, Luquin-Piudo MR, et al. Prevalencia de la enfermedad de Parkinson avanzada en pacientes tratados en los hospitales del sistema público español. Estudio PARADISE (análisis intermedio). Póster presentado en la 70. ${ }^{a}$ Reunión Anual de la Sociedad Española de Neurología (Sevilla, noviembre de 2018).

15. Martínez-Castrillo JC, Martínez-Martín P, Burgos A, Arroyo G, García N, Luquin MR, et al. Prevalence of advanced Parkinson's disease in patients treated in the hospitals of the Spanish public health care system: PARADISE Study (interim analysis) [abstract]. Mov Disord 2019; 34 (Suppl 2): abstract 1819.

16. Martínez-Castrillo JC, Martínez-Martín P, Burgos A, Arroyo G, García N, Luquin-Piudo MR, et al. Frecuencia de la enfermedad de Parkinson avanzada en hospitales del sistema público español. Estudio PARADISE. Resultados finales. Póster presentado en la $71 .^{\text {a }}$ Reunión Anual de la Sociedad Española de Neurología (Sevilla, noviembre de 2019).

17. Sociedad Española de Neurología. Mapa de recursos asistenciales neurológicos en España. Memoria 2013 de la Sociedad Española de Neurología. Presentado en la 65. ${ }^{\mathrm{a}}$ Reunión de la Sociedad Española de Neurología (noviembre de 2013).

\section{DISCREPA study: treatment of advanced Parkinson's disease and use of second-line treatments in Catalonia}

Introduction. The treatment of Parkinson's disease (PD) is complex, and the establishment of second-line therapies in advanced PD remains controversial.

Aim. To analyze the assistance of patients with PD in Catalonia, with special attention to the use of second-line therapies in advanced PD.

Subjects and methods. Online self-administered survey to neurologists in Catalonia who treated patients with PD, through the Catalan Society of Neurology.

Results. 72 neurologists who visited a monthly average of 38 PD patients (37.3\% motor complications) participated. 86\% routinely asked about motor. The main reasons for indicating second-line therapies were disability in off (83.1\%), impact of dyskinesias (76.9\%), impact of time in off (75.4\%) and time in off (73.8\%). $70 \%$ of neurologists declared limitations to establish second-line therapies: lack of resources in their hospital, lack of time to visit the patient or to perform administrative tasks and lack nursing support. Second-line therapies is not used in $72 \%$ of patients who could potentially be candidates, especially due to patient rejection (37.9\%).

Conclusions. The majority of neurologists in Catalonia who visit patients with PD routinely ask about motor complications without using specific tools. Although neurologists are well aware of the indications for the establishment of second-line therapies, the refusal of the patient, the lack of time and the lack of defined care protocols to refer patients, they can contribute to a lower use of second-line therapies in advanced PD.

Key words. Apomorphine infusion. Deep brain stimulation. Intraduodenal infusion of levodopa-carbidopa. Invasive treatment. Motor fluctuations. Parkinson's disease. 